\title{
Effects of a non-motorized transport infrastructure development in the Bucharest metropolitan area
}

\author{
M. Popa, S. Raicu, D. Costescu \& F. Rusca \\ Transport Faculty, University Politehnica of Bucharest, Romania
}

\begin{abstract}
Since 1990, in Romania and especially in Bucharest there has been a constantly growing tendency towards new expensive residential settlements located in the suburbs. Following this, even more cars have increased the road traffic towards the city centre. In the last few years, the need to extend the metropolitan area of Bucharest City has become more accepted as a solution to relocate some socio-economic activities. Bucharest is a historically developed city and there is no available space to increase the street capacity for cars and traffic. In this paper, we propose a bicycle infrastructure project to support the sustainable development of the new metropolitan area of Bucharest, and develop a model to evaluate the impacts of such a transport infrastructure on the spatial accessibility of the city centre. We reveal the importance of bicycle parking locations and their integration with urban public transport (subway and light rail). Taking into consideration a natural, native disposition of the younger population to use bicycles, in this way, we will provide quite an effective means of attracting them to non-motorized transportation as opposed to car use.
\end{abstract}

Keywords: non-motorized transportation, metropolitan area, bicycle infrastructure, spatial accessibility, public urban transit, bicycle parking.

\section{Bucharest metropolitan area and transportation}

\subsection{The situation today}

With over 2 million inhabitants (over $10 \%$ of the country's population), Bucharest is the largest town in the country and, with its multiple historic 
heritage and cultural institutions, it is one of the most important centres for tourism.

The total surface area is about 227 square $\mathrm{km}$ (urban area), with an average diameter of $21 \mathrm{~km}$. The average population density is 3600 people per sq. $\mathrm{km}$, higher than Warsaw (3400 persons per sq. km), Prague (2400 persons per sq. $\mathrm{km}$ ) or Budapest (1200 persons per sq. km).

Bucharest is located at the crossing point of two axes: one is the East-West axis, which extends from the Black Sea region to West Europe via Hungary and Austria, and the other is the North-South axis, which extends from Istanbul and Athens at the Mediterranean Sea to Russia via Ukraine.

Since 1990, there has been a constantly growing tendency towards new expensive residential settlements located in the suburbs. This is because of the natural desire to own a property (after five decades of a controlled economy); the GNP annual growth rate (between 5-6\% per year, INS [1]); the new laws concerning land property; heterogeneity of income; increasing congestion in the central area and degradation of the living environment etc.

The increase in motor-vehicle ownership in recent years is high: in 2000, there were about 240 cars per 1000 inhabitants and it is estimated that its growth is about 5\% per year, JICA [2].

Public transport (the tube, trams, trolley buses, buses) still constitutes the main mode of transport for the residents of Bucharest (Tab.1, JICA [2]) but the number of people transported by public transport is decreasing.

Table 1: $\quad$ Modal share of people movements.

\begin{tabular}{|l|r|r|}
\hline \multicolumn{1}{|c|}{ Transport mode } & Daily trips (2000) & \multicolumn{1}{|c|}{$\%$} \\
\hline Metro & 505.208 & 8,76 \\
\hline Tram & 1.109 .650 & 19,25 \\
\hline Trolley bus & 331.528 & 5,75 \\
\hline Bus & 1.015 .001 & 17,60 \\
\hline Train & 2.786 & 0,05 \\
\hline Car & 1.408 .834 & 24,44 \\
\hline Mini bus & 15.283 & 0,27 \\
\hline Cab & 233.709 & 4,05 \\
\hline Light truck & 237.114 & 4,11 \\
\hline Cycle and Motorcycle & 10.151 & 0,18 \\
\hline Walking Total & 896.336 & 15,55 \\
\hline \multicolumn{2}{|c}{} & \\
\hline
\end{tabular}

The surface public transport network is radial and ring-shaped. In fact, the new residential neighbourhoods are building in the Ilfov County area and we can see that the old peripheral villages are now becoming real satellite centres in Bucharest City, see Fig. 1. Most of these new residential areas have evolved without a strategic plan, even before the facility infrastructure has been built, Raicu [3].

The transport infrastructure in these new areas is old and poor because of the scarcity of public funds and lack of consistent strategies of residential land use. 


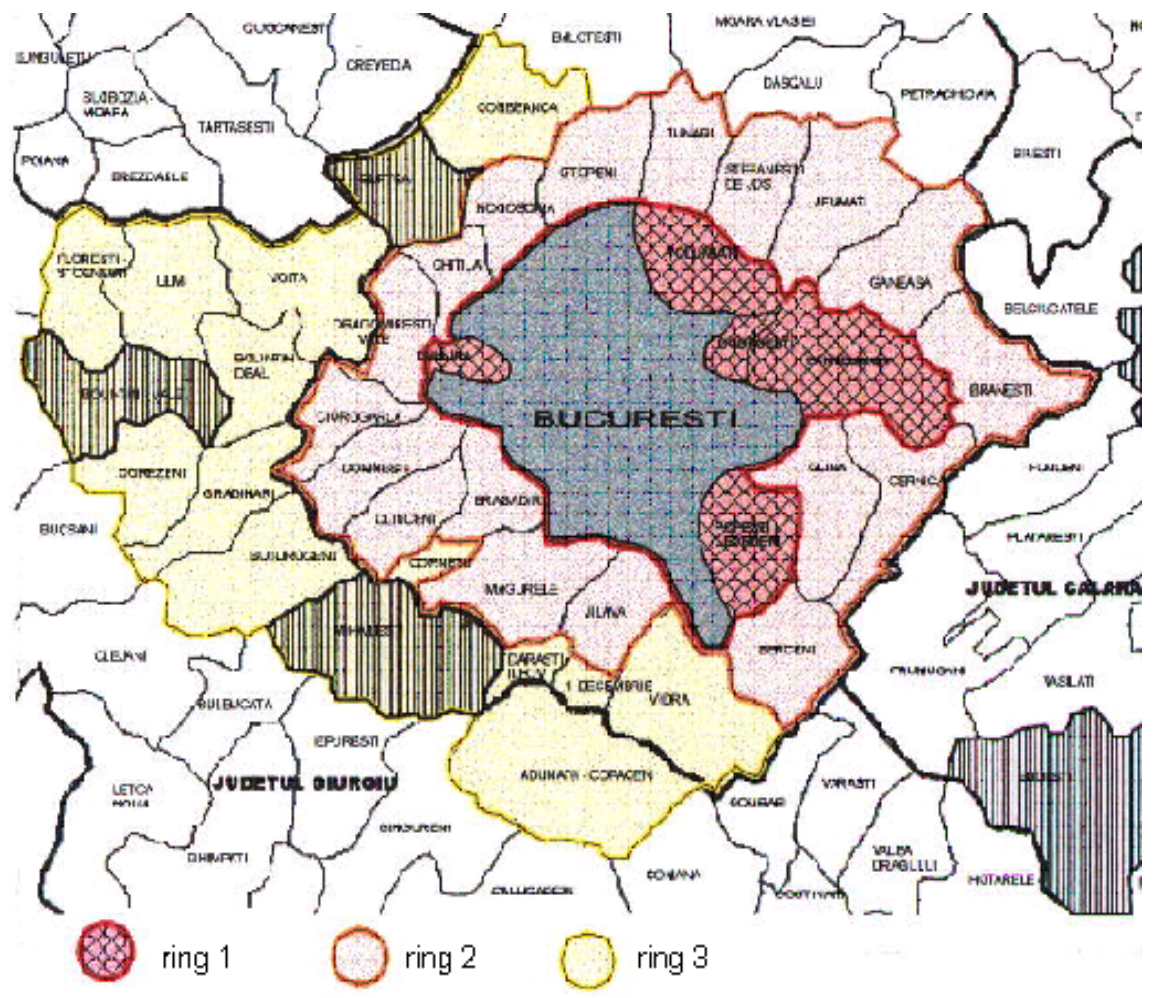

Figure 1: The new residential areas of Bucharest metropolitan space.

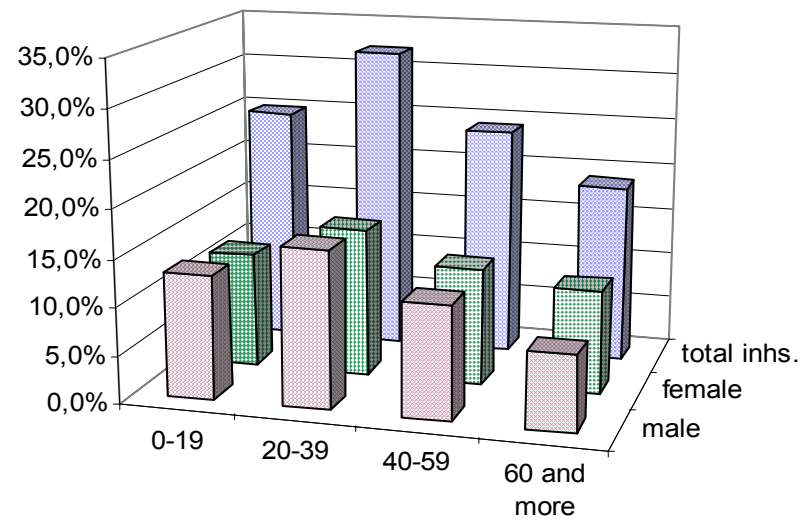

Figure 2: Age and gender structure of suburbs inhabitants.

Most of the residents from the new expensive residential areas work in Bucharest City and so they commute daily.

The age and gender structure of people from Ilfov County is that of Fig. 2, INS [1]. It is obvious that there are still many traditional residents, especially poor people, who are reliant on and trapped by public transport. 
The state public transport operator (RATB - Bucharest Transport Company) provides low quality services in these suburbs: low vehicle frequency; long usage of buses; low speeds and long vehicles headways etc., Raicu [3]. The new private operators have not changed this with their supply of so-called "microbuses".

\subsection{The new metropolitan Bucharest area project}

The National Institute of Urban Research and Development and the Bucharest City Hall prepared a General Urbanisation Plan, PUG [4] related to forecasting the general economic environment; the PUG was a main basis for "The Comprehensive Urban Transport Study of Bucharest City and its Metropolitan Area in the Republic of Romania" designed by JICA [2]. This study was selected to promote an intensive multi-centred development pattern (set-up of the suburban centre in the existing urban area to maintain compactness of the city whilst avoiding excessive concentration) inside ring 1 and ring 2 .

The other two urban development patterns analysed were:

- uncontrolled development pattern (sprawl of residential areas to surrounding farmland)

- dispersed multi-centred development pattern (set-up of new urban and industrial areas along the Outer Ring Road -ring 3, as Fig. 1 shows).

There are at least two plans of action to put into the reality this multi-centred development pattern: one is the projects supporting regional development, and the other is the administrative and social project to help the former.

Using these visions, we propose a bicycle infrastructure network (with dedicated and exclusive ways) in the new metropolitan area of Bucharest for the sustainable development of that space, Fig. 3. The most important targets of our project proposal are the younger people. The aim is to educate them in favour of non-motorized transport, and to make them aware of effects of their decisions on such infrastructure projects and on spatial accessibility of their city.

\section{The effects of the dedicated bicycle infrastructure on accessibility of the new metropolitan area}

\subsection{The model}

The spatial accessibility index defines the level of choice, taking into account both the opportunity and the modes of transport available to people. The assessment of non-motorized infrastructure development effects on the new and traditional metropolitan residents in term of spatial accessibility is a practical and a widely used approach, Smith and Halden [5].

Let us consider a circular city centre with a radial and ring-shaped network of public transport (subway and light rail). There are suburbs around the circular centre. 


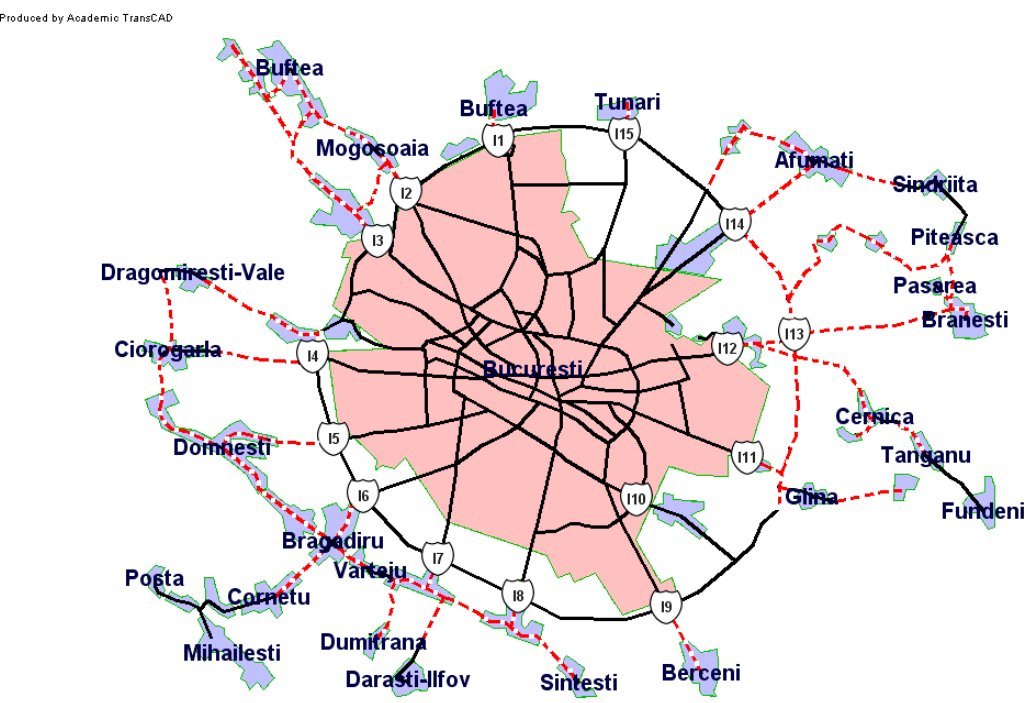

Figure 3: The new residential areas and the bicycle infrastructure linked to the public transit network (I\# is the bicycle parking space).

Let us also consider a certain place located near the centre of the city centre space, i.e. university campuses. According to the Hansen accessibility index [6], we can measure the accessibility of an entity (i.e. student), from the suburbs as follows:

$$
A=\sum_{j} E_{B, j} \cdot f_{d}\left(t_{s B}\right)
$$

where:

$f_{d}\left(t_{s B}\right)$ is the deterrence function from reaching the city centre from the suburbs, related to the travel time, having some forms,

$\sum_{j} E_{B, j}$ is the sum of all places that could possibly be chosen for the desired activity, located near to the urban centre, and in a static economic environment, this is $E_{B}$.

Without losing the generality of the model, we choose the deterrence function:

$$
f_{d}=t_{s B}^{-2}
$$

Then we have the spatial accessibility from suburbs to the city centre, when there is no dedicated or exclusive bicycle infrastructure:

$$
A^{(0)}=\frac{E_{B}}{\left(t_{C, s u b}+t_{C, B}\right)^{2}},
$$


and the spatial accessibility from the suburbs to the city centre, after the project is carried out is:

$$
A^{(1)}=\frac{E_{B}}{\left(t_{b y c}+t_{p}\right)^{2}}
$$

with:

$t_{C, s u b}, t_{C, B}$ - average travelling time by car, in the suburbs and respectively in the congested area of the city,

$t_{\text {byc }}, t_{p}$ - average travelling time by bicycle, in the suburbs and respectively the travelling time by public transport in the city area.

We define the accessibility percentage variation as follows:

$$
\Delta \alpha[\%]=\frac{A^{(1)}-A^{(0)}}{A^{(0)}} \times 100=\left(\left(\frac{t_{C, \text { sub }}+t_{C, B}}{t_{b y c}+t_{p}}\right)^{2}-1\right) \times 100
$$

If we use the average speeds and distances, respectively in the suburbs and the city area, eqn. (5) can be written as follows:

$$
\Delta \alpha=\left[\left(\frac{\frac{D_{s u b}}{D_{B}} \times \frac{1}{S_{C, s u b}}+\frac{1}{S_{C, B}}}{\frac{D_{s u b}}{D_{B}} \times \frac{1}{S_{b y c}}+\frac{1}{S_{p}}}\right)^{2}-1\right] \times 100
$$

where $S_{b y c}, S_{p}$ is the average speed by bicycle in suburbs, and respectively, by public transport in the city area,

$S_{C, \text { sub }}, S_{C, B}$ - average speed by car, in the suburbs and respectively in the congested area of the city,

$D_{\text {sub }}$ - distance of the suburbs, taking a radial way towards town,

$D_{B}$ - urban distance to the city centre (the radius of the city space),

We consider the ratio $\sigma=\frac{D_{s u b}}{D_{B}}$ like a suburb spreading, related to the centre widening. Of course, this ratio is not so near to 1 , because of the pressure of the central area (considering central area as a high density area).

From one city to another, we have a continuous domain for $\sigma$, related to many economic, social and historical variables.

Eqn. (6) can be written as follows:

$$
\Delta \alpha=\left[\left(\frac{\sigma \times \frac{1}{S_{C, s u b}}+\frac{1}{S_{C, B}}}{\sigma \times \frac{1}{S_{b y c}}+\frac{1}{S_{p}}}\right)^{2}-1\right] \times 100
$$


The accessibility variation depends on the suburbs spreading, related to urban centre space, the speeds of a car in the suburbs or the central area, and the speed of a bicycle and public transport.

\subsection{The accessibility variation related to car speed and public transport speed in urban areas}

Bucharest City is about $21 \mathrm{~km}$ in diameter. The average speed of cars at peak times is about $20 \mathrm{~km}$ per hour. All the car-ownership forecasts indicate a continuous increase in the motorization exogenous of these new residential relocations. Because of the scarcity of space in Bucharest we expect a continued decrease in car speed throughout coming years.

Fig. 4 shows the evolution of the accessibility percentage variation, taking into account this reduction and the spreading suburban areas. The suburbs are more than $3 \mathrm{~km}$ wide to the town border, which produces a negative effect on the accessibility variation. People are not willing to ride a bicycle much further than that.

As we know, any project to develop public transport in urban areas consequently increases the speed, particularly in the case of subways and light rail transport, which have exclusive ways. Nowadays, the average travel speed by public transport (as a combination of trips by subway and exclusive light rail transit) is about $30 \mathrm{~km}$ per hour. Special attention must be paid to the bicycle parking around the end station of the public transit. The parking space and its facility are quite qualitative indicators of the spatial accessibility.

In Bucharest City, under the new government, some projects have been initiated to extend the light rail transit with exclusive tracks and to complete the network ring of the tramways. We then studied the impacts of an increase in transit speed on the accessibility variation, Fig. 5 .

As it is shown, the accessibility variation to the urban central locations is increasing if the urban transit speed is increasing.

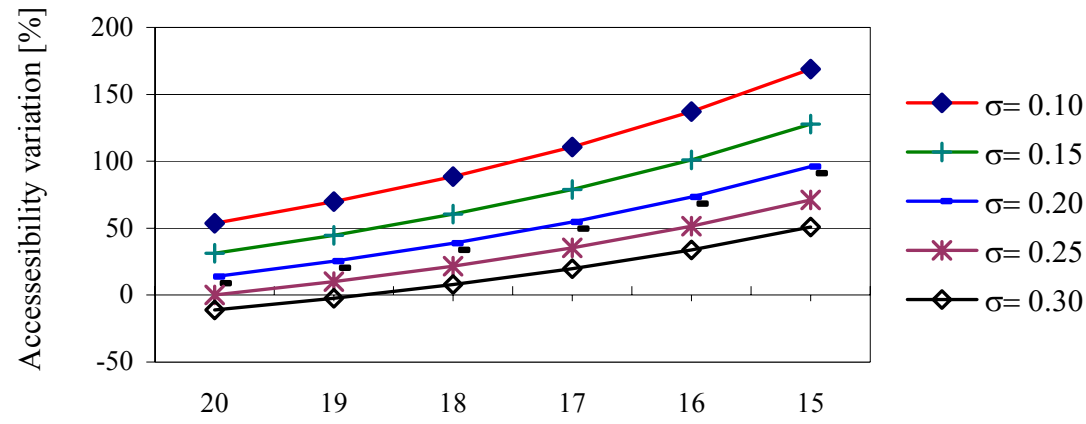

Car speed in congested urban area $[\mathrm{km} / \mathrm{h}]$

Figure 4: Accessibility variation related to car speed decreasing in congested area, and the residential area spreading. 


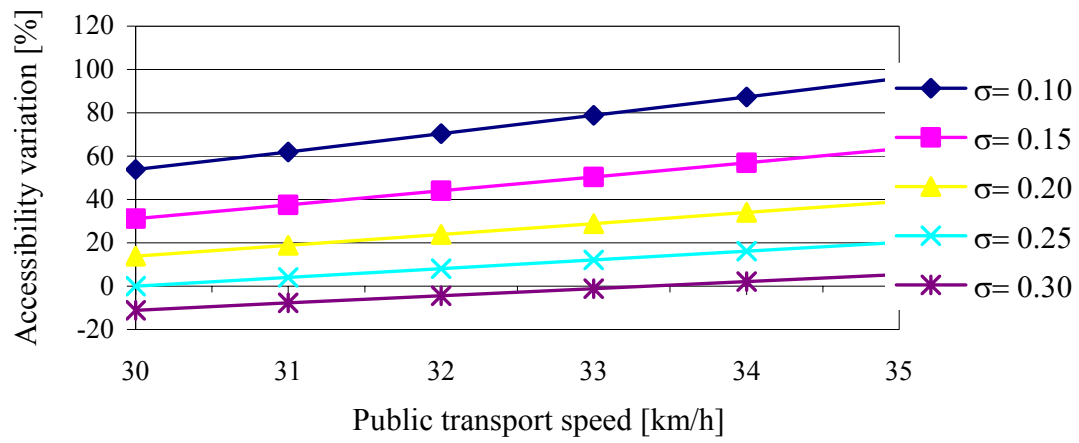

Figure 5: Accessibility percentage variation related to public transport speed increasing, and the residential area spreading.

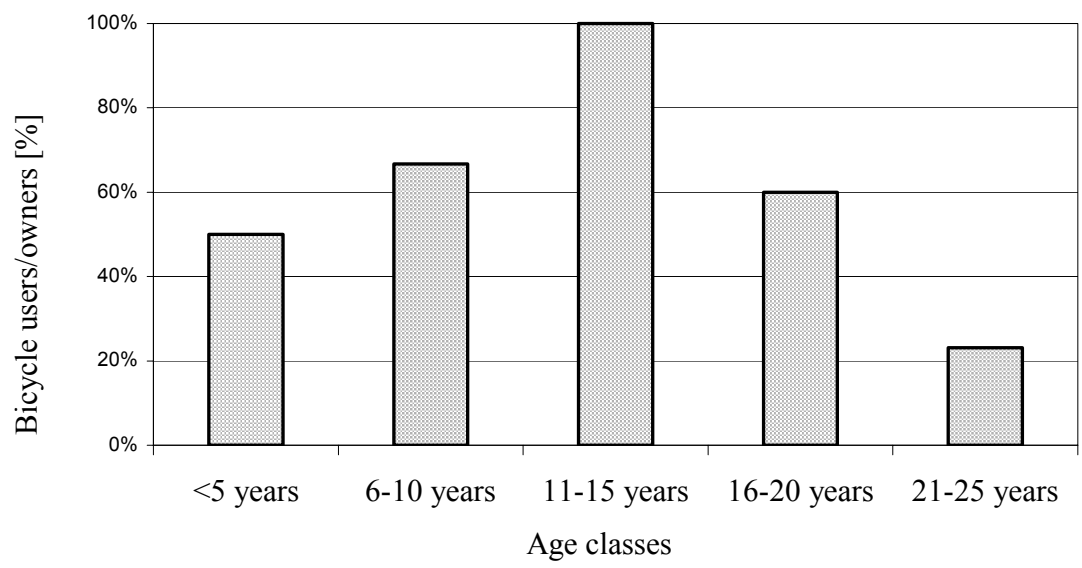

Figure 6: Rate of bicycles use related of the user's age (pilot survey results).

\section{Is there a natural tendency towards bicycle use?}

We have had the opportunity to develop research related to bicycle use of teenage and young people from schools and universities. We have some results from the pilot stage of our project (Fig.6). We are now improving our survey questions.

Our pilot survey was made to investigate 153 medium income households in a medium density area of Bucharest; the younger population was classified into five age classes, taking into account the possession of the first bicycle and bicycle usage. 
We show that there is a natural tendency to have and use a bicycle, for people around 15 years old. This tendency decreases after this age, at least because of the need for a larger space to use it (and no secure facilities to keep it).

We are also looking for the main explanations why this tendency towards bicycle usage declines after a certain age. The results will help us at least to promote the non-motorised transport infrastructure (with dedicated or exclusive ways) in the new residential metropolitan area.

\section{References}

[1] INS - National Census Institute, The Results of the Population Census 2002 (in Romanian).

[2] Japan International Cooperation Agency (JICA) The Comprehensive Urban Transport Study of Bucharest City and its Metropolitan Area in the Republic of Romania. Final Report Summary, PADECO, Bucharest, 2000, www1.pmb.ro/pmb/primar/transport_urban.pdf.

[3] Raicu, S. Researches on the developing of the transport system in areas underserved in Bucharest area (TRANSURBAN), University POLITEHNICA of Bucharest - Centre of Research, Design and Consulting in Transportation (in AMTRANS research national program, funded by the Research and Education Ministry, in Romanian), UPB, Bucharest, 2004.

[4] General Urbanistic Plan of Bucharest City -PUG, Bucharest City Hall, www4.pmb.ro/wwwt/pug/pugs.htm.

[5] Smith, D. \& Halden, D. Identifying Through Accessibility Planning how Sustainable Growth can be achieved in the Compact City: A case study of Edinburgh, www.dhc1.co.uk/features/sustainable_growth_edinburgh.html.

[6] Hansen, W.G. Haw accessibility shapes land use. Journal of the American Institute of Planners, vol.25, pp.73-76. 